\title{
Dexmedetomidine postconditioning attenuates cerebral ischemia following asphyxia cardiac arrest through down-regulation of apoptosis and neuroinflammation in rats
}

\section{Dan Fan ( $\sim$ fandan1976@163.com)}

Sichuan Provincial People's Hospital: Sichuan Academy of Medical Sciences and Sichuan People's Hospital https://orcid.org/0000-0003-3245-7702

\section{GuangQian Li}

University of Electronic Science and Technology of China

\section{Lei Qian}

University of Electronic Science and Technology of China

\section{Pan Gu}

University of Electronic Science and Technology of China

\section{Research article}

Keywords: Dexmedetomidine, post-conditioning, cerebral ischemia, asphyxia cardiac arrest, apoptosis, neuroinflammation

Posted Date: December 21st, 2020

DOI: https://doi.org/10.21203/rs.3.rs-130943/v1

License: (c) (i) This work is licensed under a Creative Commons Attribution 4.0 International License.

Read Full License 


\title{
Dexmedetomidine post-conditioning attenuates cerebral
} ischemia following asphyxia cardiac arrest through

\section{down-regulation of apoptosis and neuroinflammation in rats}

\author{
Guangqian $\mathrm{Li}^{1 \#}$, Lei Qian ${ }^{1 \#}, \operatorname{Pan~Gu}^{1}$, Dan Fan ${ }^{1,2^{*}}$
}

1. School of Medicine, University of Electronic Science and Technology of China, Chengdu, China

2. Department of Anesthesiology, Sichuan Academy of Medical Sciences and Sichuan Provincial People's Hospital, Chengdu, China

\# Contributed equally to this work and should be considered co-first authors *Corresponding Author: Department of anesthesiology, Sichuan Academy of Medical Sciences and Sichuan Provincial People's Hospital, No. 32 West Second Section, First Ring Road, Chengdu, Sichuan, China, 610072; E-mail: fandan1976@163.com.

\begin{abstract}
Background and Purpose Neuroprotection strategies after cardiac arrest (CA)/cardiopulmonary resuscitation (CPR) remain key areas of basic and clinical research. This study was designed to investigate the neuroprotective effects of dexmedetomidine following resuscitation and potential mechanisms.
\end{abstract}

Methods Anesthetized rats underwent 6-minute asphyxia-based cardiac arrest and resuscitation, after which the experimental group received a single intravenous dose of dexmedetomidine $(25 \mu \mathrm{g} / \mathrm{kg})$. Neurological outcomes and ataxia were assessed after the return of spontaneous circulation. The serum levels and brain expression of inflammation markers was examined, and apoptotic cells were quantified by TUNEL 
staining.

Results: Neuroprotection was enhanced by dexmedetomidine post-conditioning after the return of spontaneous circulation. This enhancement was characterized by the promotion of survival, neurological function scores and coordination. In addition, dexmedetomidine post-conditioning attenuated the serum levels of the pro-inflammatory cytokine tumor necrosis factor (TNF)- $\alpha$ at $2 \mathrm{~h}$, as well as interleukin (IL)-6 at 2, 24, and 48h. TUNEL staining showed that the number of apoptotic cells in the dexmedetomidine post-conditioning group was significantly reduced compared with in the control group. Further western blot analysis indicated that dexmedetomidine markedly reduced the levels of caspase-3 and nuclear factor-kappa $\mathrm{B}(\mathrm{NF}-\kappa \mathrm{B})$ in the brain.

Conclusions: Dexmedetomidine post-conditioning had a neuroprotective effect against cerebral injury following asphyxia-induced cardiac arrest and improved the survival rate. The mechanism was associated with the down-regulation of apoptosis and neuroinflammation.

Keywords: Dexmedetomidine, post-conditioning, cerebral ischemia, asphyxia cardiac arrest, apoptosis, neuroinflammation

\section{Background}

Cardiac arrests $(\mathrm{CA})$ is a leading cause of death worldwide ${ }^{1,2}$. Although recent developments in cardiopulmonary resuscitation (CPR) techniques and post-resuscitation care have improved the chances of survival, there are still high rates of death and disability following the restoration of spontaneous circulation (ROSC) ${ }^{3}$. 
Survivors of CA suffer from painful sequelae, including anoxic brain injury, myocardial dysfunction and the systemic ischemia/reperfusion response, which are described as post-CA syndrome, whereby cerebral injury is the main reason of death and disability after $\operatorname{ROSC}^{3-5}$. The brain consumes the largest amount of oxygen of all organs, and is highly susceptible to disruptions of blood flow. Sudden cardiac arrest induces complete cerebral ischemia, followed by a cascade of detrimental events that can lead to immediate and delayed brain damage, including excitotoxicity, oxidative stress and inflammation ${ }^{5-7}$. What's more, cardiopulmonary resuscitation can lead to reperfusion injury, which may exacerbate brain damage. Neurons in affected areas of brain undergo delayed cell death, which disrupts the shaping of neural circuits and ultimately leads to both motor and cognitive dysfunction. Because of the high incidence of CA and the complex etiology of cerebral ischemia-reperfusion injury, it is urgent to find a therapeutic strategy to attenuate post-CA brain injury.

Dexmedetomidine (Dex) is a specific agonist of $\alpha 2$-adrenergic receptors that has been used as a sedative in intensive care since 1999, and also as an adjuvant to reduce the dosage of other anesthetics ${ }^{8,9}$. Recently, a growing body of research found neuroprotective effects of Dex in different experimental models of cerebral injury ${ }^{10-12}$. While the uncontrolled, systemic inflammatory response is a critical cause of brain injury following ischemia/reperfusion (I/R), it was found that Dex can reduce the expression of inflammatory factors after brain I/R injury, which may be related with the inhibition of the toll-like receptor-4//NF- $\mathrm{B}(\mathrm{TLR}-4 / \mathrm{NF}-\kappa \mathrm{B})$ pathway $^{12,13}$. On the other hand, overproduction of free radicals and destruction of natural antioxidant 
function is another cause of I/R-induced brain injury. Additionally, studies have shown that Dex can attenuate oxidative stress ${ }^{14-16}$. What's more, the antiapoptotic effect of Dex is likely caused by an improvement of mitochondrial function and the inhibition of neuronal autophagy ${ }^{17}$. However, whether these pharmacological effects of Dex could alleviate post-CA brain damage was not known, and the underlying mechanism have not been fully understood.

The present study used a rat model of CA/CPR to investigate the protective effects of Dex against brain injury and investigate the potential mechanisms.

\section{Methods}

Animals: All the experiments were approved by the Animal Care and Use Committee of the Sichuan Academy of Medical Sciences and Sichuan Provincial People's Hospital and the animals received humane care in compliance with the Guide for the Care and Use of Laboratory Animals published by the US National Institutes of Health (NIH Publication No. 85-23, revised 1996). A total of 30 male Sprague-Dawley rats, weighing 350-450g, were obtained from the Chengdu Dashuo Experimental Animal Centre of Sichuan, China. The animals were housed at a constant temperature $\left(23 \pm 1^{\circ} \mathrm{C}\right)$ on a $12 \mathrm{~h}$ light/dark cycle with free access to food and water, two rats were placed in per cage. These housing environments were maintained until the animals were sacrificed under deeply anesthesia with isoflurane for brain tissue harvest .

Asphyxial cardiac arrest model: The rat asphyxial CA model was established as reported previously ${ }^{18}$, with minor modifications as follows. Each rat was anesthetized 
using an intraperitoneal injection of pentobarbital sodium solution $(45 \mathrm{mg} / \mathrm{kg})$ and mechanically ventilated (respiratory frequency $60 \mathrm{bpm}$, tidal volume $8 \mathrm{ml} / \mathrm{kg}$ ) using a Harvard Ventilator (Model 683, Harvard Apparatus, Holliston, MA, USA). A rectal probe was inserted to monitor the body temperature of the rats, which was maintained at $36^{\circ} \mathrm{C} \pm 1{ }^{\circ} \mathrm{C}$ using a heating pad. The right femoral artery and vein were exposed. A venous indwelling catheter $(24 \mathrm{G})$ filled with heparin saline was placed in the femoral artery and connected to a pressure transducer (Powerlab 16/30, AD-Instruments, Australia) to monitor the arterial blood pressure. Another $24 \mathrm{G}$ venous indwelling catheter was placed into the femoral vein for fluid infusion. The rats were monitored for at least 10 minutes to record the baseline. After muscle relaxation with cisatracurium besilate $(0.2 \mathrm{mg} / \mathrm{kg})$, asphyxial CA was induced by clamping the tube in the trachea and stopping the ventilator. CA was defined as a systolic blood pressure $(\mathrm{SBP})<25 \mathrm{mmHg}$. Six minutes after CA, CPR and mechanical ventilation were initiated. External anterior to posterior chest compressions at a depth of $1 / 3$ diameter of the rat thorax were carried out at a frequency of 200/min. During resuscitation, epinephrine $(0.01 \mathrm{mg} / \mathrm{kg}), 5 \%$ sodium bicarbonate $(0.36 \mathrm{ml} / \mathrm{kg})$ and $0.9 \%$ saline $(0.5 \mathrm{ml})$ were injected into femoral vein through the indwelling catheter. Restoration of spontaneous circulation (ROSC) was defined as the return of spontaneous sinus rhythm, with SBP > $60 \mathrm{mmHg}$, which was maintained for at least 10 minutes. Spontaneous respiration was carefully monitored every 5 minutes. The rats were weaned from the ventilator after spontaneous respiration totally recovered. Finally, the venous indwelling catheters were withdrawn from the right femoral artery and vein. 
Experimental protocol: All rats were randomly assigned to three groups: 1) sham operation group (sham, $\mathrm{n}=6$ ); 2) CA/CPR without any treatment (control, $n=12$ ); 3) CA/CPR plus post-treatment with dexmedetomidine (Dex, $n=12$ ). The tail of each rat was marked by different color markers according group design, and the cage was labeled the group name.The sham rats went through all the operational procedures except for cardiac arrest and CPR. After restoration of spontaneous circulation, rats in the Dex and control groups received a single intravenous injection of Dex $(25 \mathrm{mg} / \mathrm{kg}$, Hengrui Medicine, Jiangsu, China) or the same volume of saline, respectively. Dex or saline was pumped into the vein using a micro-infusion pump for about 30min.

Evaluation of neurological deficits: Neurological examination was performed by an investigator who was blinded to the experimental design using the neurological deficit scores (NDS), which ranges from 80 (best) to 0 (brain dead) and includes a subscore of general behavioral deficit: consciousness as normal, stuporous or unresponsive and arousal with eye opening and respiration as normal, abnormal (hypo or hyperventilation) or absent. The NDS of the surviving rats was assessed at 24, 48 and 72h after CA/CPR. The brainstem function sub-scores were assessed as follows: (1) olfaction, as response to the smell of food; (2) vision, as head movement toward light; presence of (3) pupillary light reflex; (4) corneal reflex; (5) startle reflex; (6) response to whisker stimulation and (7) swallowing of liquids or solids. The sub-score for motor assessment included strength testing as normal, abnormal (either stiff or weak) and absence of movement. The sensory assessment sub-score included response to limb pinch as brisk withdrawal, weak or abnormal response (extension or flexion 
posture) and no response. The motor behavior sub-score was assessed based on gait coordination as normal, abnormal or none. Balance on a beam was judged as normal if the rat could cross a $2 \mathrm{~cm}$ wide and $1 \mathrm{~m}$ long beam suspended $0.5 \mathrm{~m}$ above the floor; abnormal if the rat attempts and does not continue or stays momentarily and falls. The assessment was scored as absent if the rat falls off immediately upon placing on the beam. Other evaluated behavioral reflex sub-scores include: (1) righting reflex (animal placed on its back and is able to correct to upright position); (2) turning alley (the animal is made to walk and turn back at the end of a $15 \mathrm{~cm}$ by $0.5 \mathrm{~m}$ alley); (3) visual placing (the animal is lifted and is able to visually orient itself to objects and depth); and (4) negative geotaxis (animal is placed on its back on a plane angled at $45^{\circ}$ and the animal corrects itself and moves upward on the incline). The last subscore assesses the occurrence of seizures (convulsive or non-convulsive).

Rotarod test: The rotarod test is designed to evaluate the motor coordination and balance ability of rats. It includes adaptation training and a test process. Before CA surgery, the rats in each group were trained continuously for 3 days. The rotating bar fatigue meter was set to $4 \mathrm{rpm}$. The animals were trained 3 times a day for at least 15min each time, and the interval between the two training sessions was at least 15min. The final testing was performed 5 days after ROSC. All surviving rats were individually placed on the rotating rod, the rotation speed was increased from 4rpm to 40rpm within 260 s and the time from the beginning to the fall of the rat recorded. The test was repeated three times, and the average amount of time until falling was taken as the final result. 
Serum levels of inflammatory factors: Retro-orbital blood samples (0.8-1.2 mL) were collected at 2, 24, and $48 \mathrm{~h}$ after ROSC, the serum was separated by centrifugation at $12000 \mathrm{~g}$ for $10 \mathrm{~min}$ and immediately analyzed or stored at $-80^{\circ} \mathrm{C}$. The levels of IL-6 and TNF-a were analyzed using commercial ELISA kits (R\&D systems) according to the manufacturer's instructions. All measurements were carried out in duplicate.

Western blot analysis: Brain tissue was collected at 5 days after ROSC, and total protein lysates were prepared using lysis buffer (Thermo Scientific, Rockford, IL, USA) containing protease inhibitors cocktail (Sigma-Aldrich) and phosSTOP phosphatase Inhibitor Cocktail (Roche, Nutley, NJ, USA). The BCA assay kit (Thermo Fisher Scientific, USA) was used to measure the protein concentration. Samples comprising $20 \mu \mathrm{g}$ total protein per lane was separated by SDS-PAGE and then transferred to a PVDF membrane. The membranes were blocked with 5\% non-fat milk for about $1 \mathrm{~h}$ at room temperature and incubated with the following primary antibodies overnight at $4^{\circ} \mathrm{C}$ : rabbit polyclonal anti-caspase-3 antibody (1:1000; Cell

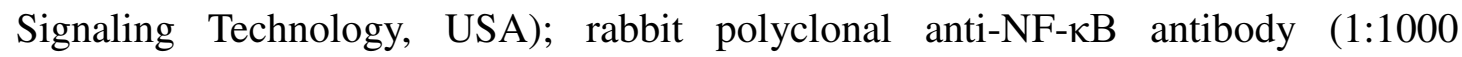
Protein-tech, China); $\quad \alpha$-tubulin (1:5000; Protein-tech, China,). After incubation with secondary antibodies, the immunoreactive bands were developed using enhanced chemiluminescence reagents (Pierce, IL, USA) and was visualized using GeneSnap software version 7.08. The protein amounts were quantified densitometrically using Image $\mathbf{J}$ software and normalized to the density of $\alpha$-tubulin in the same sample. The results of rats from the different experimental groups were then normalized to the 
mean values of the corresponding control animals.

TUNEL staining: The TUNEL assay was performed using the Apoptosis \& Cell Death Assay kit (Merck Millipore, USA) according to the manufacturer's instructions. Briefly, brain sections were incubated with proteinase $\mathrm{K}$ at room temperature for $30 \mathrm{~min}$, and then incubated with the TUNEL reagent at $37^{\circ} \mathrm{C}$ for $1 \mathrm{~h}$. The sections were then washed with PBS and counter-stained with 4',6-diamidino-2-phenylindole (DAPI). Fluorescence images were captured using a fluorescence microscope at $40 \times$ magnification. The results were quantified as apoptotic index (AI\%), which was defined as the ratio of positive apoptotic cells to all cells in the same field of view.

Statistical analysis SPSS 20.0 software (IBM Corp., USA) was used for statistical analysis. The survival curves were determined using the Kaplan-Meier method and compared with the log-rank test. Repeated test results are presented as means $\pm \mathrm{SD}$ and differences with $P$-values $<0.05$ were considered statistically significant. Charts were rendered using GraphPad Prism 6.0 software.

\section{Results}

\section{Dex improves survival and hemodynamics after CA/CPR}

Application of Dex significantly improved the survival at 5 days after CA/CPR. The survival rates of the rats in the Control and Dex groups were 50 and 66.7\%, respectively. All five rats in the Sham group survived. The log - rank test showed that the differences were statistically significant $(\mathrm{p}<0.05)$ (Fig. 1A). Compared with the basal level, the mean arterial pressure (MAPs) of the control and Dex groups decreased significantly after resuscitation, and notable decreases were observed at 
15-25 min after ROSC $(P<0.05)$ (Fig. 1B). After resuscitation, the heart rate (HR) decreased in both groups, with no marked difference between the two groups $(P>$ 0.05), and both returned to the baseline $1 \mathrm{~h}$ after ROSC (Fig. 1C).

\section{Dex attenuated the impairment of nerve and motor function following CA/CPR}

The NDS was evaluated at 24,48 and $72 \mathrm{~h}$ after CA/CPR. In the sham group, the NDS score was approximately 80 at all the time points. After CA, the NDS of the control group was obviously decreased compared with the sham group. Treatment with Dex markedly attenuated the neurological deficit score (Fig. 2A).

In addition to neurological function disorders, CA/CPR can also damage motor function. In this study, the rotarod test was used to assess the ataxia of rats at 5 days after CA. After CA/CPR, the surviving rats in the Control group showed significantly poorer scores in all indicators, including the average rotarod speed (Fig. 2B), the total time on the rotarod (Fig. 2C), rotation speed at fall (Fig. 2D) and the total walking distance (Fig. 2D). As can be seen in the corresponding figures, the application of Dex effectively attenuated the neurological impairment.

\section{Dex reduced the expression of pro-inflammatory factors following CA/CPR}

To evaluate the anti-inflammatory effect of Dex following CA/CPR operation, the serum levels of IL- $1 \beta$ and TNF- $\alpha$, as well as the expression of NF- $\kappa \mathrm{B}$ in brain tissues were evaluated at 2, 24 and 48 hours after CA/CPR. Compared with the sham group, the serum levels of IL-1 $\beta$ and TNF- $\alpha$ (Figs. 3A and B) and the brain tissue

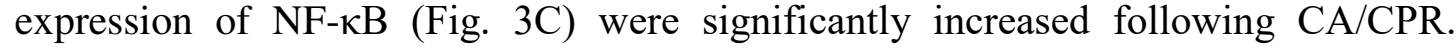
However, application of Dex after resuscitation decreased the production of IL-1 $\beta$ and 
TNF- $\alpha$, while also blocking the increase of NF- $\kappa B$ in the brain (Fig. 3).

\section{Dex inhibited the expression of proteins related to neuronal apoptosis following}

\section{CA/CPR.}

To understand whether the protective effect of Dex is related to neuronal apoptosis, TUNEL staining and western blot analyses were performed to assess the percentage of apoptotic neurons and expression of the proapoptotic factor caspase-3. According to TUNEL staining, there were few positive cells in the sham group, but their percentage increased after CA/CPR (Fig. 4A). Furthermore, Dex significantly decreased the number of TUNEL-positive neurons (Fig. 4A). The proportion of TUNEL-positive neurons was done by cell counting in a single field of view, which showed that the apoptosis index of $\mathrm{CA} / \mathrm{CPR}$ rats was significantly increased, while Dex effectively blocked this increase (Fig. 4B). In addition, treatment with Dex significantly reduced the expression of caspase-3 (Figs. 4C and D).

\section{Discussion}

In this study, we explored the potential effects of Dex on the survival and neurological function of rats after CA/CPR. The main findings of this study include: 1) treatment with Dex after resuscitation improved the survival rate of rats after CA/CPR; 2) Dex ameliorated the CA/CPR-induced neurological deficits; and 3) Dex may exert its protective effect by reducing inflammation and inhibiting apoptosis.

$\mathrm{CA} / \mathrm{CPR}$ induces systemic ischemia-reperfusion (I/R) injury, which activates the immune system and causes a systemic inflammatory response. During CA/CPR, leukocytes, macrophages and tissue-resident immune cells recognize the injury and 
release primary cytokines, which in turn induce the recruitment and activation of 244 leukocytes, largely amplifying the inflammatory response ${ }^{19,20}$. The brain uses 20 245 percent of the body's oxygen and calories ${ }^{21}$, and can therefore suffer severe damage 246 due to CA. As the crucial resident immune cells of the central nervous system( CNS), 247 microglia express various cytokine receptors, recognizing IL-1 and TNF- $\alpha$, among 248 many others. Consequently, microglia will be over-activated after I/R injury and 249 release excess pro-inflammatory cytokines, impairing neural function ${ }^{22,23}$. A growing 250 body of evidence suggests that inflammation is crucial for the pathogenesis of 251 neurological deficits after $\mathrm{CA} / \mathrm{CPR}^{24-27}$. In this study, we found that the serum levels 252 of TNF- $\alpha$ and IL-6 were significantly increased after ROSC in the CPR group. The 253 levels of pro-inflammatory factors peaked at $2 \mathrm{~h}$ and returned to baseline levels within 254 48h after ROSC. Treatment with Dex attenuated the increase of TNF- $\alpha$ and IL-6, 255 improving the neurological outcomes. CA/CPR can cause sympathetic nerve 256 over-excitation, which may exacerbate further inflammation and cause significant 257 neurotoxicity ${ }^{28,29}$. Dex is a highly specific agonist of the $\alpha 2$-adrenergic receptor, and 258 is commonly used as an adjuvant anesthetic. Furthermore, several studies 259 demonstrated the anti-inflammatory effect of Dex in different models. For instance, 260 Dex was found to significantly improve cognitive function after carotid 261 endarterectomy by inhibiting CNS inflammation ${ }^{30}$. Moreover, Zheng and colleagues 262 showed that Dex inhibited CNS neuroinflammation after traumatic brain injury (TBI), 263 and reduced the expression of the nucleotide-binding oligomerization domain 264 (NOD)-like receptor family pyrin domain containing 3(NLRP3) inflammasome ${ }^{31}$. 
Additionally, previous research suggests that post-treatment with Dex could attenuate early brain injury (EBI) induced by subarachnoid hemorrhage (SAH), and that it exerts its protective effect by inhibiting the activation of the TLR4/NF- $\kappa \mathrm{B}$ pathway, the release of pro-inflammatory cytokines and the expression of the NLRP3 inflammasome ${ }^{32}$. NF- $\kappa \mathrm{B}$ is a transcription factor that regulates many genes, especially inflammation-related cytokines. Dex was shown to reduce the expression of Toll-like receptor 4 and suppress the activation of NF- $\kappa B$ by interacting with the $\alpha-2$ receptor ${ }^{33}$. The results of this study are in agreement with this theory, and we found that treatment with Dex can effectively suppress the phosphorylation of NF- $\kappa \mathrm{B}$ following CA/CPR.

Recently, there has been increasing evidenc that neuronal apoptosis is also a key reason for CNS dysfunction after $\mathrm{I} / \mathrm{R}^{5,34}$. Apoptotic programmed cell death is mainly

277 induced by specific proteins such as Apaf-1, as well as proteins in the Bcl-2 and 278 caspase families ${ }^{35}$. In mammalian cells, apoptosis is triggered by two main pathways, called the intrinsic pathway and the extrinsic pathway, which both converge in the activation of caspase- $3^{3637}$. Neuronal apoptosis is initiated by the cleavage of caspase-3 and results in DNA breakdown, degradation of cytoskeletal components, 282 and the production of apoptotic particles, which are finally engulfed by phagocytic 283 cells $^{38}$. CA/CPR compromises the integrity of the blood-brain barrier and activates microglial cells, resulting in the release of inflammatory mediators and reactive 285 oxygen species. These toxic chemicals inhibit the production of neurotrophic factors 286 and disturb the effective communication between brain cells ${ }^{39}$. In this study, TUNNEL 
staining showed a significant increase in the number of apoptotic neurons following

288 CA/CPR, which could be effectively alleviated by Dex. The level of cleaved 289 caspase-3 is universally recognized as a specific marker of apoptosis ${ }^{17}$. Our findings 290 indicated that Dex decreased the concentration of cleaved caspase-3 in brain tissues, 291 which was in accordance with the results of TUNEL-staining. Previous research 292 demonstrated that Dex exerts its antiapoptotic effect by reducing the levels of 293 pro-inflammatory factors and $\operatorname{ROS}^{40,41}$. In addition, Dex improves the survival of 294 neurons by activating the brain-derived neurotrophic factor/ tropomyosin-related 295 kinase B(BDNF/TrkB )pathway ${ }^{42,43}$.

296 The current study also has some inadequacies and limitations. For example, we were not able to use a concentration gradient to confirm the best therapeutic dose due to limitations in the number of experimental animals that can be handled. What's more, exploration of the possibility of combination therapy, such as dexmedetomidine 300 combined with hypothermia therapy, is still awaiting further research.

\section{Conclusions}

Our findings indicate that post-resuscitation treatment with dexmedetomidine has a significant neuroprotective effect and attenuates neurological disorders following CA/CPR. The potential mechanism through which dexmedetomidine exerts its protective effects is likely related to the suppression of neuroinflammation and promotion of neuron survival by inhibiting apoptosis.

\section{Abbreviations}

AI: apoptotic index; BDNF: brain-derived neurotrophic factor; CA: cardiac arrest; 
CNS: central nervous system; CPR: cardiacpulmonary resuscitation;

DAPI: 4',6-diamidino-2-phenylindole; Dex: dexmedetomidine; EBI: early brain injury; HR: heart rate; IL: interleukin; I/R: ischemia reperfusion; MAPs: mean arterial pressure; NDS: neurological deficit scores; NF-кB: nuclear factor-kappa B;

NIH: National Institutes of Health; NOD: nucleotide-binding oligomerization domain; NRLP3: (NOD)-like receptor family pyrin domain containing 3; ROSC: restoration of spontaneous circulation; SBP: systolic blood pressure; SAH: subarachnoid hemorrhage; TBI: traumatic brain injure; TNF- $\alpha$ :tumor necrosis factor alpha; TrkB: tropomyosin-related kinase B; TLR-4: toll-like receptor 4.

\section{Declarations}

\section{Ethics approval and consent to participate}

This study was approved by the Animal Care and Use Committee of the Sichuan Academy of Medical Sciences and Sichuan Provincial People's Hospital. And the animals received humane care in compliance with the Guide for the Care and Use of Laboratory Animals published by the US National Institutes of Health (NIH Publication No. 85-23, revised 1996).

\section{Consent to publish}

Not Applicable.

\section{Availability of data and materials}

All data generated or analyzed during this study are included in this published article and supporting data can be obtained from the corresponding author upon reasonable request. 


\section{Competing interests}

332 The authors declare they have no competing interests.

\section{$333 \quad$ Funding}

334 This work was Funded by the National Natural Science Foundation of China 335 (No.81500933) and the Sichuan science and technology department research projects, 336 China (No. 2015SZ0080). The Funder is Dr. DF, the corresponding author which 337 designed the experiment and revised the manuscript.

\section{Authors' contributions}

339 LQ and DF contributed to the concept and design of the study. GQL and LQ and PG 340 collected and analyzed the data. GQL and LQ wrote the original draft. DF critically 341 reviewed and revised the manuscript. All authors have read and approved the final 342 manuscript.

\section{Acknowledgements}

344 Not applicable.

\section{References}

B.46 Zhang, S. Sudden cardiac death in China: current status and future perspectives. Europace :

347 European pacing, arrhythmias, and cardiac electrophysiology: journal of the working groups

348 on cardiac pacing, arrhythmias, and cardiac cellular electrophysiology of the European

349 Society of Cardiology17 Suppl 2, ii14-18 (2015).

3.50 Kragholm, K. et al. Bystander Efforts and 1-Year Outcomes in Out-of-Hospital Cardiac Arrest.

351 New England Journal of Medicine376, 1737-1747 (2017).

3.52 Stock, D., Jacob, B., Chan, V., Colantonio, A. \& Cullen, N. Change in Function Over 

Inpatient Rehabilitation After Hypoxic Ischemic Brain Injury: A Population-Wide Cohort Study. Archives of physical medicine and rehabilitation100, 1640-1647 (2019).

Stub, D., Bernard, S., Duffy, S.J. \& Kaye, D.M. Post cardiac arrest syndrome: a review of therapeutic strategies. Circulation123, 1428-1435 (2011).

Zheng, J.H. et al. PD98059 protects the brain against mitochondrial-mediated apoptosis and autophagy in a cardiac arrest rat model. Life sciences232, 116618 (2019).

Ma, Q. et al. Annexin A1 Bioactive Peptide Promotes Resolution of Neuroinflammation in a Rat Model of Exsanguinating Cardiac Arrest Treated by Emergency Preservation and Resuscitation. Front Neurosci13, 608 (2019).

Gaudier-Diaz, M.M., Haines, A.H., Zhang, N. \& DeVries, A.C. Social influences on microglial reactivity and neuronal damage after cardiac arrest/cardiopulmonary resuscitation. Physiol Behav194, 437-449 (2018).

Ma, D., Rajakumaraswamy, N. \& Maze, M. alpha2-Adrenoceptor agonists: shedding light on neuroprotection? British medical bulletin71, 77-92 (2004).

Huupponen, E. et al. Electroencephalogram spindle activity during dexmedetomidine sedation and physiological sleep. Acta anaesthesiologica Scandinavica52, 289-294 (2008).

Liao, Z. et al. Both JNK and P38 MAPK pathways participate in the protection by dexmedetomidine against isoflurane-induced neuroapoptosis in the hippocampus of neonatal rats. Brain Res Bull107, 69-78 (2014).

Zeng, X., Wang, H., Xing, X., Wang, Q. \& Li, W. Dexmedetomidine Protects against Transient Global Cerebral Ischemia/Reperfusion Induced Oxidative Stress and Inflammation in Diabetic Rats. PLoS One11, e0151620 (2016). 
B7.5 Chen, Z., Qiu, P.Y. \& Ma, C.G. Dexmedetomidine preconditioning protects against retinal

376 ischemia/reperfusion injury and inhibits inflammation response via toll-like receptor 4 (TLR4)

377 pathway. Biomedicine \& pharmacotherapy = Biomedecine \& pharmacotherapie $\mathbf{9 3}$,

$378 \quad 1018-1024(2017)$

B3.9 Kim, E. et al. Dexmedetomidine confers neuroprotection against transient global cerebral

380 ischemia/reperfusion injury in rats by inhibiting inflammation through inactivation of the

381 TLR-4/NF-kappaB pathway. Neurosci Lett649, 20-27 (2017).

B\$.2 Rodriguez-Gonzalez, R. et al. Neuroprotective effects of dexmedetomidine conditioning

383 strategies: Evidences from an in vitro model of cerebral ischemia. Life sciences144, 162-169

384 (2016).

B8.5 J. Huang, Q.J. Dexmedetomidine Protects Against Neurological Dysfunction in a Mouse

386 Intracerebral Hemorrhage Model by Inhibiting Mitochondrial Dysfunction-Derived Oxidative

387 Stress. Journal of Stroke and Cerebrovascular Diseases (2019).

B88.8 X. H. Chen, D.T.C., X. M. Huang, et al. Dexmedetomidine protects against chemical

389 hypoxia-induced neurotoxicity in differentiated PC12 cells via inhibition of NADPH oxidase

390 2-mediated oxidative stress. Neurotoxicity research35(1): 139-149 (2019).

BØ.1 Wu, G.J. et al. Protection of Dexmedetomidine Against Ischemia/Reperfusion-Induced

392 Apoptotic Insults to Neuronal Cells Occurs Via an Intrinsic Mitochondria-Dependent Pathway.

393 Journal of cellular biochemistry118, 2635-2644 (2017).

B8.4 Delayed, s.h.r.n.d.a. \& rats, a.c.a.i. Delayed, spontaneous hypothermia reduces neuronal

395 damage after asphyxial cardiac arrest in rats. Crit Care Med (2000).

B9.6 Shen, H., Kreisel, D. \& Goldstein, D.R. Processes of Sterile Inflammation. The Journal of 
Immunology191, 2857-2863 (2013).

Ortega-Gomez, A., Perretti, M. \& Soehnlein, O. Resolution of inflammation: an integrated view. EMBO molecular medicine5, 661-674 (2013).

Magistretti, Pierre J. \& Allaman, I. A Cellular Perspective on Brain Energy Metabolism and Functional Imaging. Neuron86, 883-901 (2015).

Block, M.L., Zecca, L. \& Hong, J.S. Microglia-mediated neurotoxicity: uncovering the molecular mechanisms. Nat Rev Neurosci8, 57-69 (2007).

Cunningham, C. Microglia and neurodegeneration: the role of systemic inflammation. Glia61, 71-90 (2013).

Liang, L. et al. Xuezhikang improves the outcomes of cardiopulmonary resuscitation in rats by suppressing the inflammation response through TLR4/NF-kappaB pathway. Biomedicine $\&$ pharmacotherapy $=$ Biomedecine \& pharmacotherapie114, 108817 (2019).

Wang, J. et al. Inhibition of Soluble Epoxide Hydrolase after Cardiac Arrest/Cardiopulmonary Resuscitation Induces a Neuroprotective Phenotype in Activated Microglia and Improves Neuronal Survival. Journal of Cerebral Blood Flow \& Metabolism33, 1574-1581 (2013).

Wei, X. et al. Hydrogen Sulfide Inhalation Improves Neurological Outcome via NF-\&\#954;B-Mediated Inflammatory Pathway in a Rat Model of Cardiac Arrest and Resuscitation. Cellular Physiology and Biochemistry36, 1527-1538 (2015).

Taguchi, N., Nakayama, S. \& Tanaka, M. Single administration of soluble epoxide hydrolase inhibitor suppresses neuroinflammation and improves neuronal damage after cardiac arrest in mice. Neuroscience research111, 56-63 (2016).

Willemze, R.A., Luyer, M.D., Buurman, W.A. \& de Jonge, W.J. Neural reflex pathways in 
intestinal inflammation: hypotheses to viable therapy. Nature reviews. Gastroenterology \& hepatology12, 353-362 (2015).

Pereira, M.R. \& Leite, P.E.C. The Involvement of Parasympathetic and Sympathetic Nerve in the Inflammatory Reflex. Journal of Cellular Physiology231, 1862-1869 (2016).

Ge, Y. et al. Dexmedetomidine improves cognition after carotid endarterectomy by inhibiting cerebral inflammation and enhancing brain-derived neurotrophic factor expression. $J$ Int Med Res47, 2471-2482 (2019).

Zheng, B. et al. Administration of Dexmedetomidine inhibited NLRP3 inflammasome and microglial cell activities in hippocampus of traumatic brain injury rats. Bioscience Reports38, BSR20180892 (2018).

Yin, D. et al. Dexmedetomidine attenuated early brain injury in rats with subarachnoid haemorrhage by suppressing the inflammatory response: The TLR4/NF-kappaB pathway and the NLRP3 inflammasome may be involved in the mechanism. Brain Res1698, 1-10 (2018). Huang, Y. et al. Perineural Dexmedetomidine Attenuates Inflammation in Rat Sciatic Nerve via the NF-kB Pathway. International Journal of Molecular Sciences15, 4049-4059 (2014). Han, Z.W. et al. GPER agonist G1 suppresses neuronal apoptosis mediated by endoplasmic reticulum stress after cerebral ischemia/reperfusion injury. Neural Regen Res14, 1221-1229 (2019).

Elmore, S. Apoptosis: a review of programmed cell death. Toxicologic pathology35, 495-516 (2007).

Fricker, M., Tolkovsky, A.M., Borutaite, V., Coleman, M. \& Brown, G.C. Neuronal Cell Death. Physiological reviews $\mathbf{9 8 ,}$ 813-880 (2018). 
Yuan, J.Y. \& Yankner, B.A. Apoptosis in the nervous system. Nature407, 802-809 (2000).

Li, P. et al. Oxidative stress and DNA damage after cerebral ischemia: Potential therapeutic targets to repair the genome and improve stroke recovery. Neuropharmacology134, 208-217 (2018).

Feng, X. et al. Electroacupuncture ameliorates cognitive impairment through inhibition of NF-kappaB-mediated neuronal cell apoptosis in cerebral ischemia-reperfusion injured rats. Mol Med Rep7, 1516-1522 (2013).

Chen, L. et al. Protective effect of dexmedetomidine against diabetic hyperglycemia-exacerbated cerebral ischemia/reperfusion injury: An in vivo and in vitro study. Life sciences, 116553 (2019).

Qian, X.L. et al. Dexmedetomidine improves early postoperative cognitive dysfunction in aged mice. European journal of pharmacology746, 206-212 (2015).

Chiu, K.M. et al. Dexmedetomidine protects neurons from kainic acid-induced excitotoxicity by activating BDNF signaling. Neurochemistry international129, 104493 (2019).

Tu, Y. et al. Dexmedetomidine attenuates the neurotoxicity of propofol toward primary hippocampal neurons in vitro via Erk1/2/CREB/BDNF signaling pathways. Drug design, development and therapy13, 695-706 (2019).

\section{Appendices}

Figure 1 Dex improved the survival and hemodynamics of rats following cardiac arrest and cardiopulmonary resuscitation (CA/CPR). (A) Survival of the Sham, Control and Dex groups. (B and C) Changes in the mean arterial pressures (MAP) and heart rate (HR) in the Control and Dex groups after CA/CPR. Data are expressed as 
the means \pm SD. $(\mathrm{n}=6-12) . * P<0.05$

464 Figure 2 Dex attenuated the impairment of neurological and motor function after

465 CA/CPR. (A), The neurological deficit score evaluated at 24, 48 and 72 hours after ROSC. (B-D) Rotarod performance tests were conducted at 5 days after ROSC. The results are shown as the average speed of the rotarod (B), the total time of walking on the rotarod (C), the rotation speed at falling (D) and the total distance of rat the walking on the rotarod (E). The data are presented as the means \pm SD. $(n=6)$. $* * * p<0.001 ; * * p<0.01 ; * p<0.05$

Figure 3 Dex reduced the expression of pro-inflammatory cytokines after CA/CPR.

472 The serum levels of IL-1 $\beta(\mathrm{A})$ and TNF- $\alpha(\mathrm{B})$ at 2, 24 and 48 hours after resuscitation. (C-D) The protein levels of NF- $\mathrm{kB}$ in brain tissues. The data are presented as the means \pm SD. $(\mathrm{n}=6) * p<0.05$

Figure 4 Dex inhibits the apoptosis of neurons after CA/CPR. CA/CPR-induced apoptosis, as assessed by TUNEL staining. (A) The quantitative analysis results of TUNEL staining. (B) Treatment with Dex decreased the levels of cleaved caspase-3.

479 the means \pm SD. $(\mathrm{n}=4-6) . * P<0.05$ 


\section{Figures}
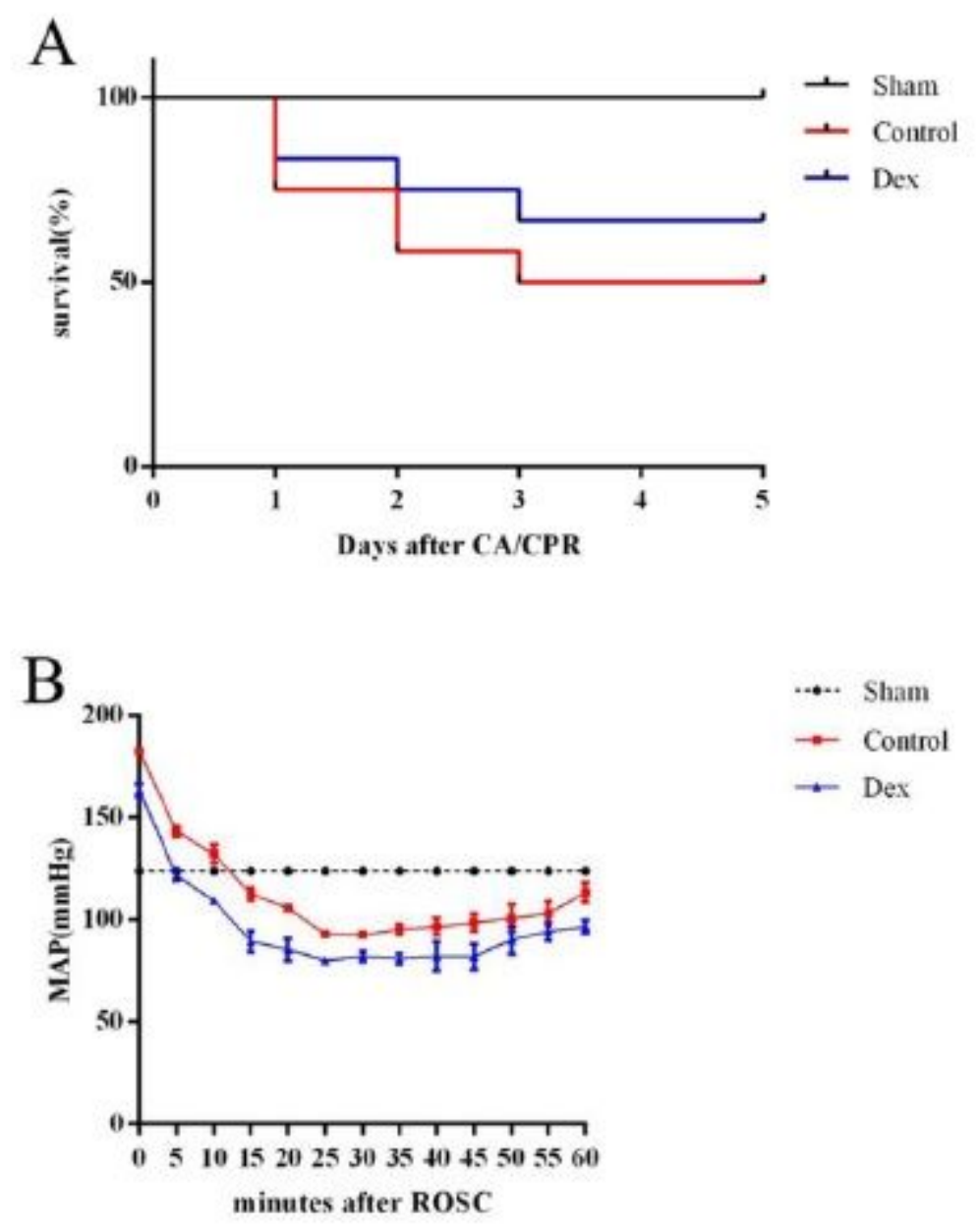

.... Sham

$\rightarrow$ Control

$\rightarrow$ Dex

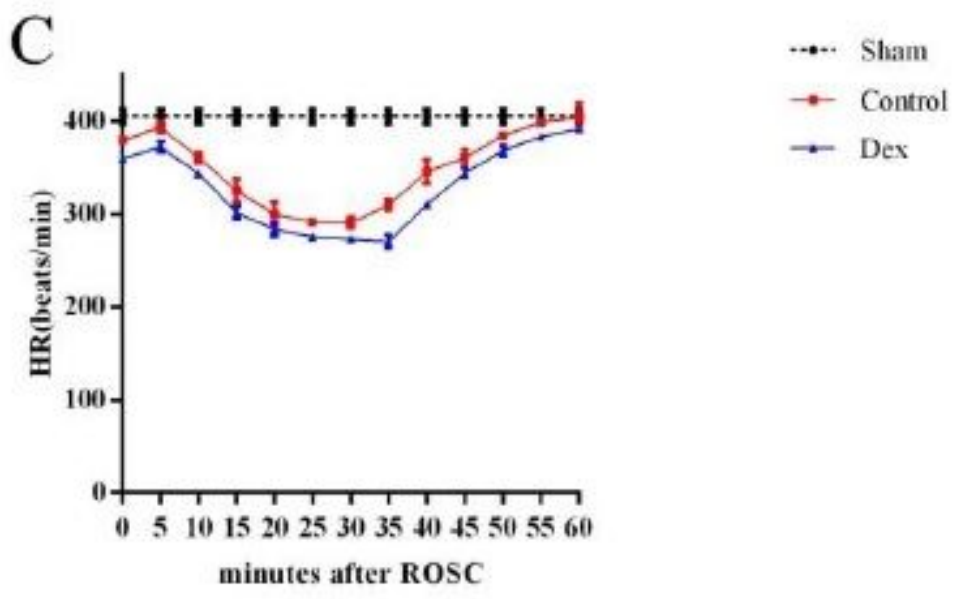

\section{Figure 1}

Dex improve $d$ the survival and hemodynamics of rats following cardiac arrest and cardiopulmonary resuscitation (CA/CPR). (A) Survival of the Sham, 460 Control and Dex groups. (B and C) Changes in the mean arterial pressures (MAP) and 461 heart rate (HR) in the Control and Dex groups after CA/CPR. Data are expressed as the mean the meanss \pm SD. $\pm S D .(n=6(n=6-12) 12) . . * * P P<0.05<0.05$ 
A
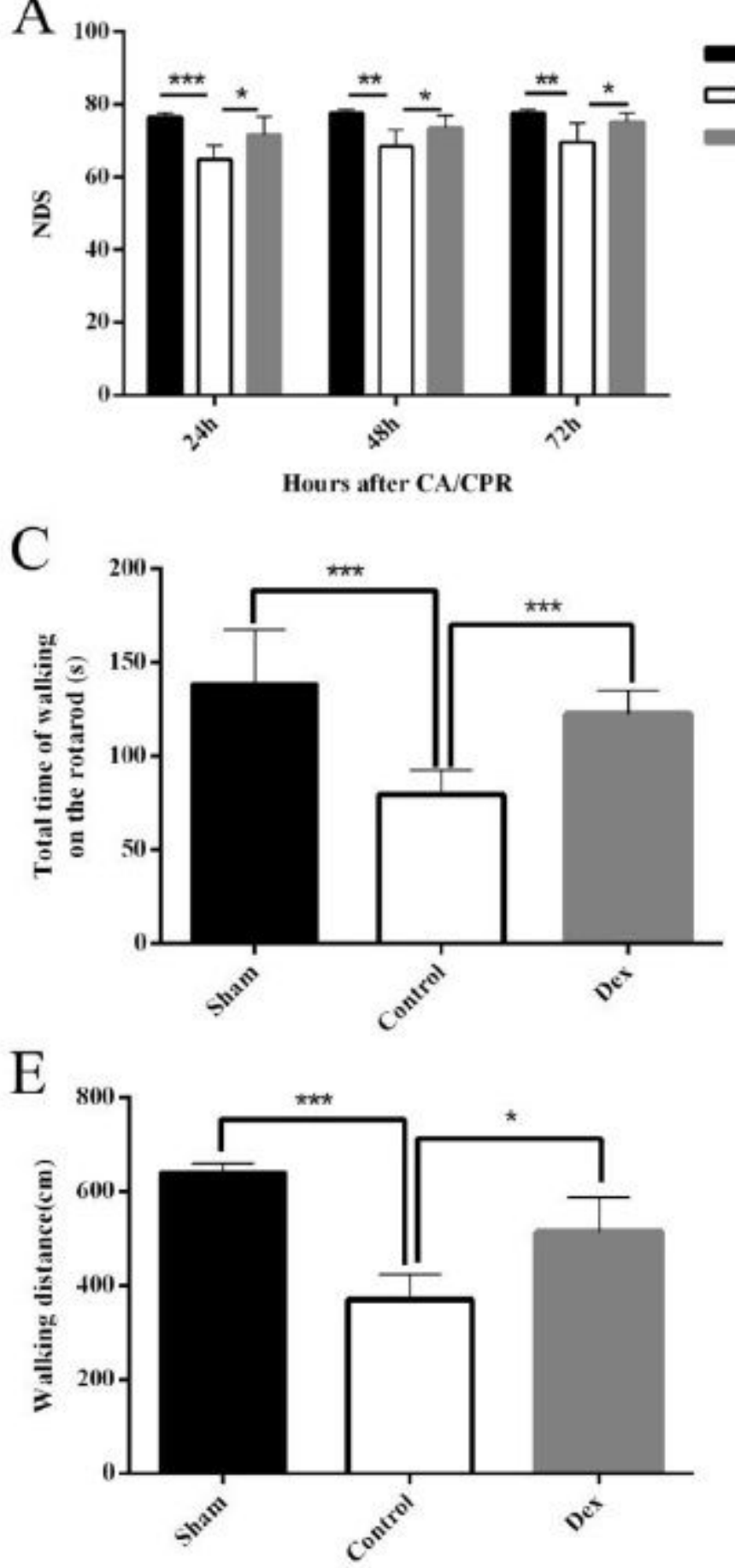
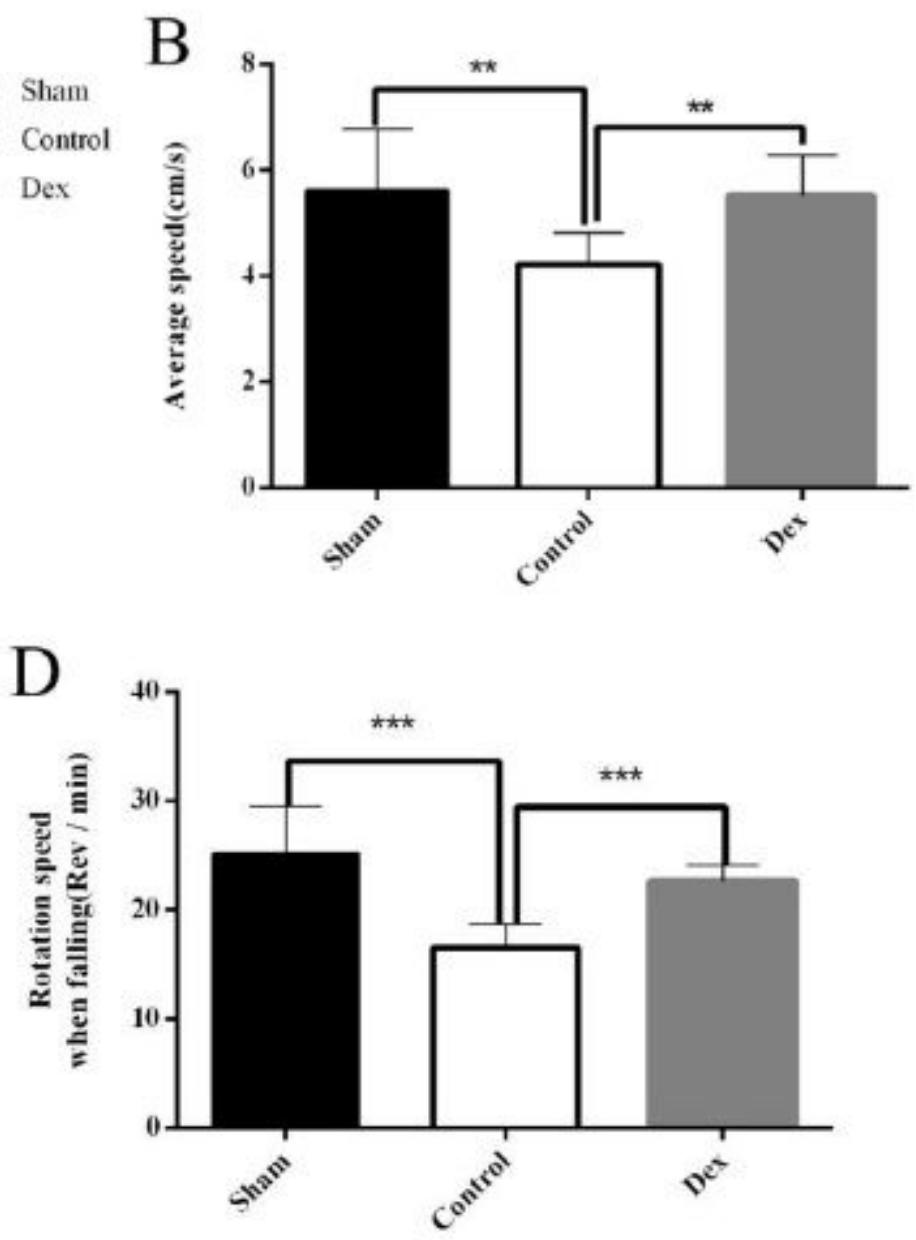

Figure 2

Dex attenuated the impairment of neurological and motor functi on after CA/CPR. A )), T he neurological deficit score evaluated at 24, 48 and 72 hours after ROSC. (B D) Rotarod performance tests were conducted at 5 days after ROSC. The result s are shown as the average speed of the ro ta rod (B), the total time of walking on the rotarod $(C)$, the ro ta tion speed at falling (D) and the total distance of rat the 468 walking on the ro ta rod $(E)$. The $d$ at a are presented as the mean $S \pm S D . .(n=p<0.001 ; p<0.01 ; p$ $<0.05$ 

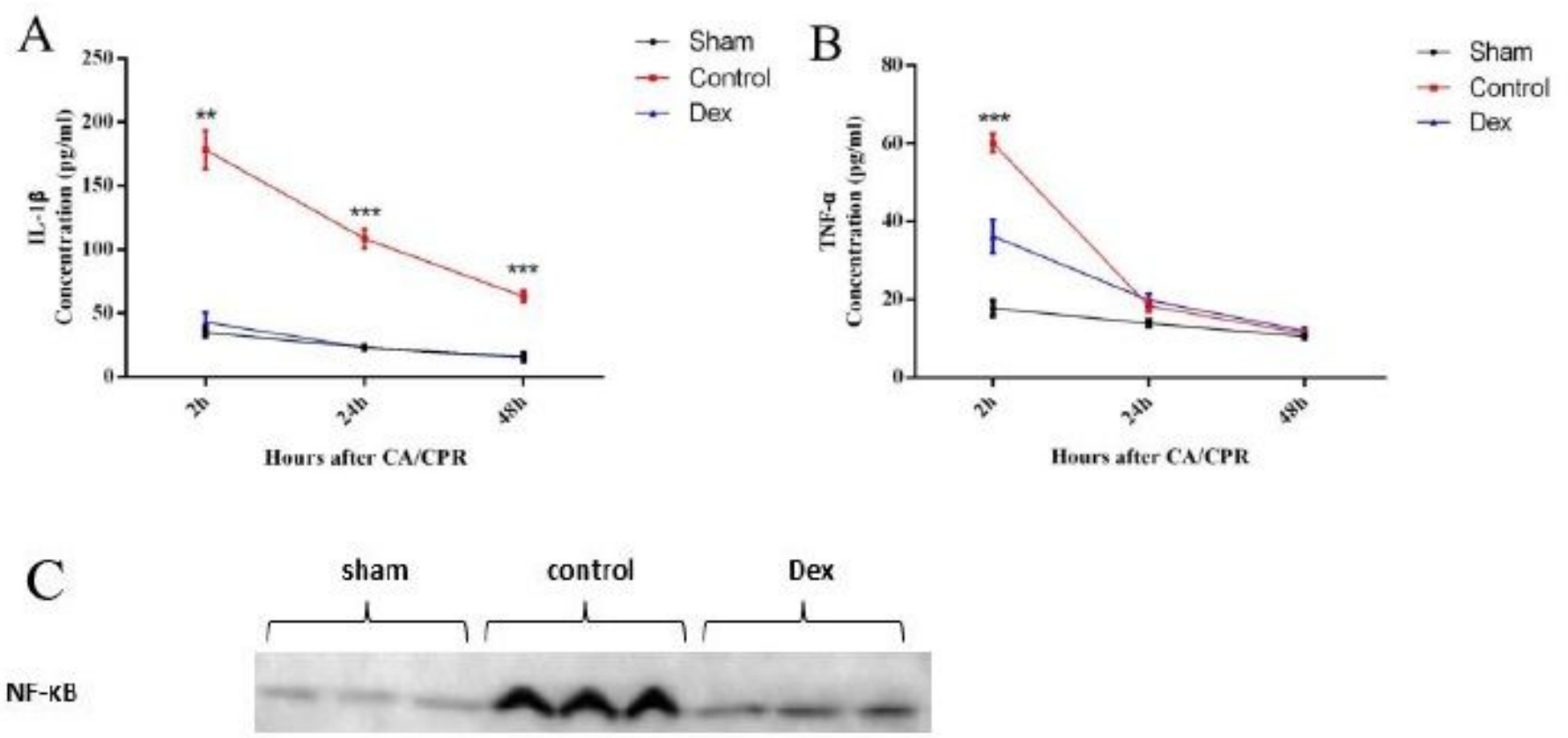

$\alpha$-tubulin
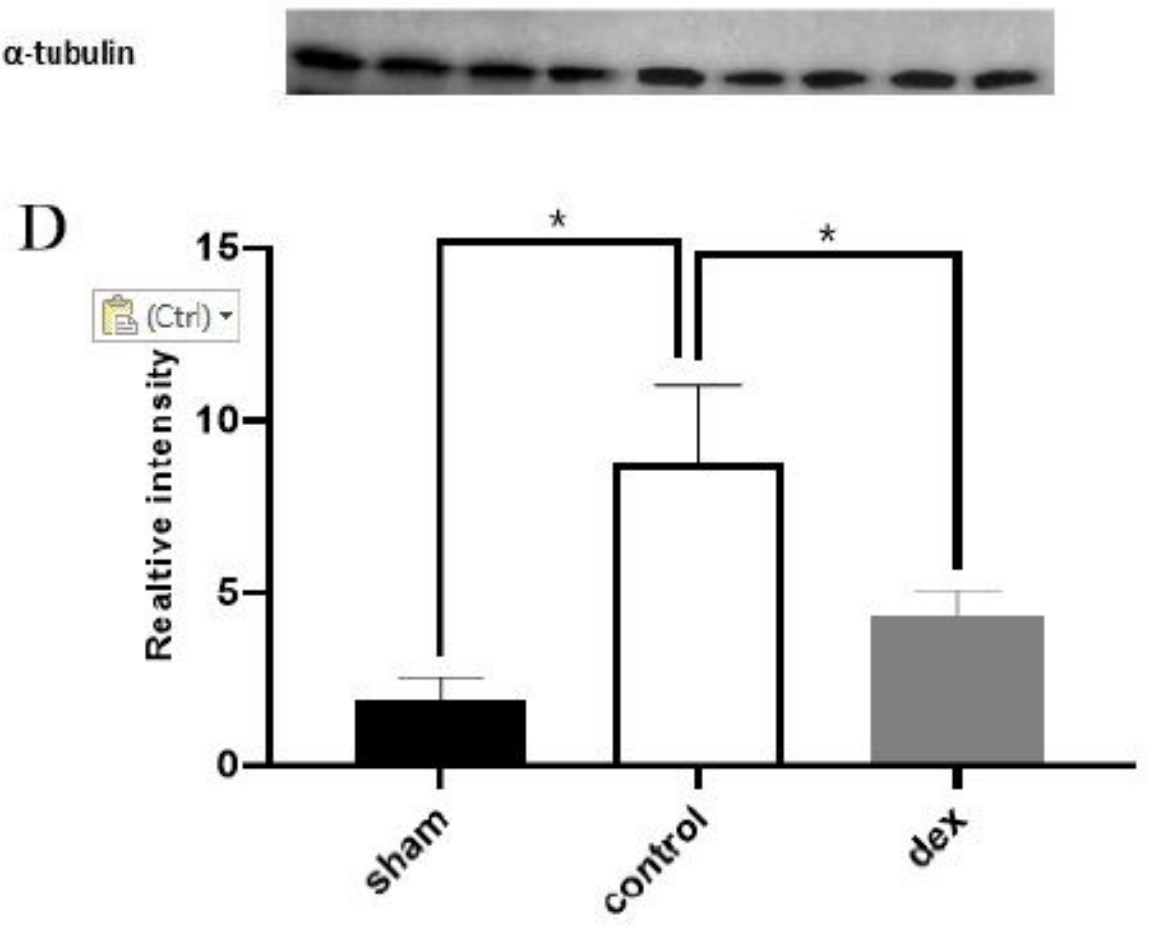

Figure 3

$D$ ex reduced the expression of pro inflammat ory cytokines after CA/CPR. T he serum level s of IL $1 \beta$ A and TNF a (at 2, 24 and 48 hours after resuscitation. C D T he protein level s of NF KB in brain tissues . The $d$ at a are presented as the mean $s \pm S D$..(n=p<0.05 


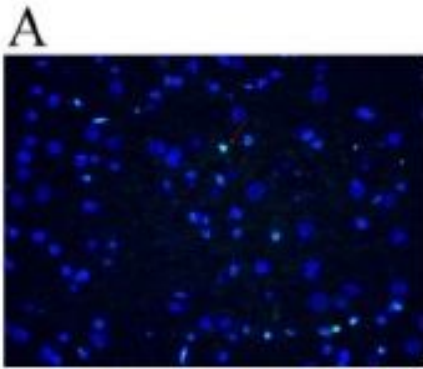

Sham

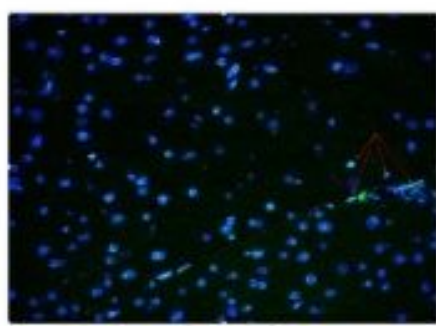

Control

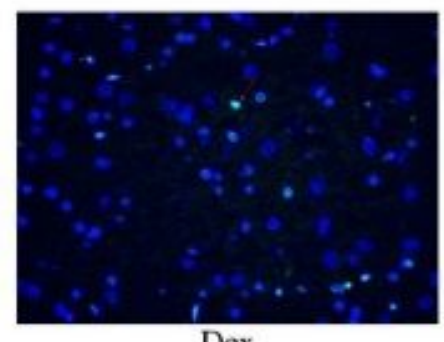

Dex

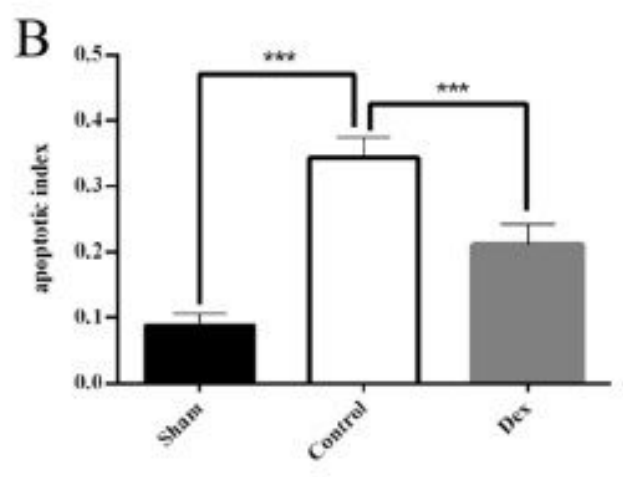

cleaved caspase-3

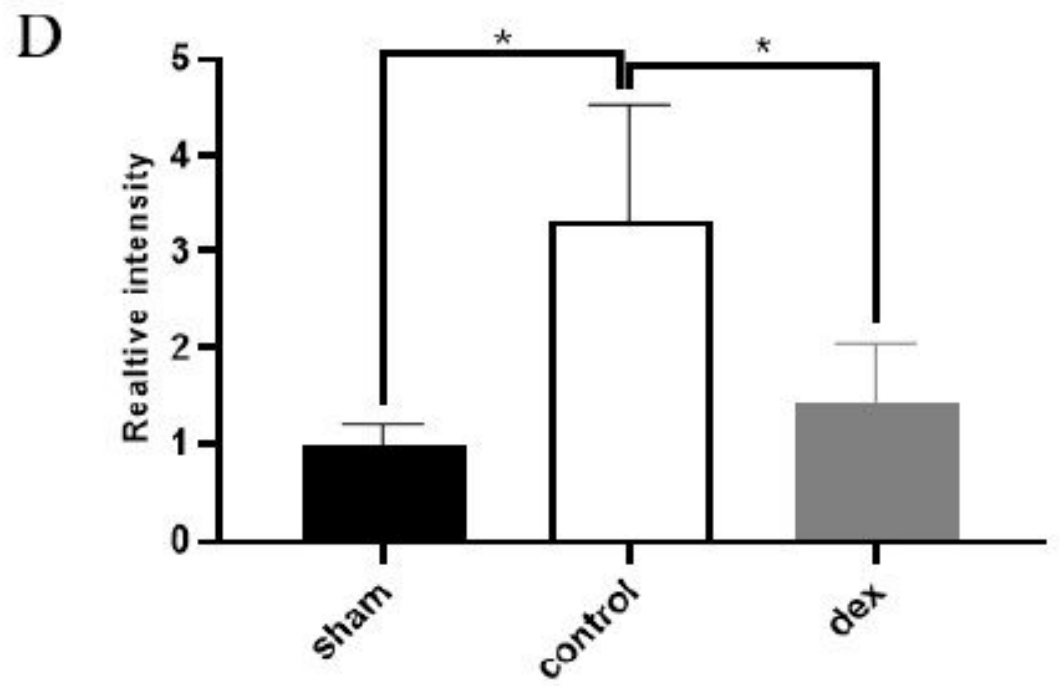

Figure 4

Dex inhibits the apoptosis of neurons after CA/CPR. CA/CPR induced apoptosis, as assessed by TUNEL staining (A) The quantitative analysis results of TUNEL staining (B) Treatment with Dex decreased the levels of cleaved caspase 3. (D) Western blot analysis results, normalized to a tubulin. The data are expressed as the mean $s \pm S D(n=46) . P<0.05$ 


\section{Supplementary Files}

This is a list of supplementary files associated with this preprint. Click to download.

- AuthorChecklistFull.pdf

- Caspase3.png

- WBOriginalResults.pdf

- renamed4e86e.png 